\title{
A!
}

This is an electronic reprint of the original article.

This reprint may differ from the original in pagination and typographic detail.

Volovik, G.E.; Zubkov, M.A.

\section{Mirror as polaron with internal degrees of freedom}

Published in:

Physical Review D

DOI:

10.1103/PhysRevD.90.087702

Published: 01/01/2014

Document Version

Publisher's PDF, also known as Version of record

Please cite the original version:

Volovik, G. E., \& Zubkov, M. A. (2014). Mirror as polaron with internal degrees of freedom. Physical Review D, 90(8), 1-5. [087702]. https://doi.org/10.1103/PhysRevD.90.087702

This material is protected by copyright and other intellectual property rights, and duplication or sale of all or part of any of the repository collections is not permitted, except that material may be duplicated by you for your research use or educational purposes in electronic or print form. You must obtain permission for any other use. Electronic or print copies may not be offered, whether for sale or otherwise to anyone who is not an authorised user. 


\title{
Mirror as polaron with internal degrees of freedom
}

\author{
G. E. Volovik \\ Low Temperature Laboratory, Aalto University, P.O. Box 15100, FI-00076 Aalto, \\ Finland and Landau Institute for Theoretical Physics, RAS, \\ Kosygina 2, 119334 Moscow, Russia \\ M. A. Zubkov \\ University of Western Ontario, London, Ontario, Canada N6A $5 B 7$ and ITEP, \\ B.Cheremushkinskaya 25, Moscow 117259, Russia \\ (Received 19 May 2014; published 27 October 2014)
}

\begin{abstract}
We consider the model suggested by Wang and Unruh [Phys. Rev. D 89, 085009 (2014)] for the $1+1$-dimensional mirror moving in the quantum vacuum. We consider the relation of this model to the problem of the polaron - the electron moving in the vacuum of the quantum field of phonons. We introduce the field-theoretical model of such a mirror. It contains the multicomponent spinor field interacting with the scalar field. We discuss the source of the logarithmic divergence in the mirror mass and its relation to the problem of the divergencies in vacuum energy.

DOI: 10.1103/PhysRevD.90.087702

PACS numbers: 42.50.Lc, 98.80.Es
\end{abstract}

\section{INTRODUCTION}

The authors of [1] consider the mirror with an internal harmonic oscillator coupled to a scalar field in $1+1$ dimensions. It is found that the effective mass (the rest energy) of such a composite mirror is infinite (logarithmically divergent) due to the vacuum fluctuations of the scalar field. This system is considered in [1] as the counter example to the generally accepted statement that the vacuum energy matters only when taking gravity into account (otherwise one can only measure the energy differences). It is argued that in the given system the infinities like that of the vacuum energy do matter.

First of all, we disagree with the above-mentioned generally accepted statement. In condensed matter physics the vacuum energy density participates in the thermodynamics of the system together with the energy density of matter. That is why we expect that the vacuum energy density matters both in the Universe with gravity and in the Universe without gravity (see the latter case in Ref. [2]). When one tries to calculate the vacuum energy density or the ground state energy density of condensed matter systems summing the energies of fluctuations, one obtains the divergent sum with the ultraviolet (UV) cutoff determined by the high-energy scale. In particle physics such estimate suggests a huge value of the cosmological constant. However, the condensed matter systems, where the microscopic physics (the analog of the trans-Planckian physics) is known, demonstrate that the divergent highenergy contributions from zero point energies of quantum field are cancelled by the microscopic (trans-Planckian) degrees of freedom due to the thermodynamic identities. If the same pattern is applicable to particle physics, and to the Universe as a whole, then we are to treat the overall volume of the Universe as variable. The variation over volume demonstrates that the equilibrium is achieved when the thermodynamic potential vanishes, which is equal to the cosmological constant (see [3] and recent review [4]). Within this approach it is natural to assume that we live near the equilibrium. Therefore, the total vacuum energy density with all contributions taken into account should be very close to zero. This provides the cancellation of the main UV divergent terms in the known low energy theory by something coming from the unknown high energy theory. As a result only a small fraction of the vacuum energy density remains that is comparable with the observed value of the cosmological constant. This cosmological constant gives rise to the accelerated expansion of the Universe. The latter expansion should be understood as the small deviation of the Unverse from equilibrium.

Next, we analyze the model discussed in Ref. [1]. This model operates with the massive oscillator moving along its world line and interacting with the scalar field. The world line fluctuates, and its fluctuations are to be defined in accordance with the action functional of the moving oscillator. We reformulate this model in a standard way in terms of the quantum field of the moving oscillator. This allows to calculate the renormalization of mass for the given object due to the interaction with the scalar field using the standard perturbation theory. The obtained infinity is the result of the unrealistic approximation made in the model: the mirror is assumed to be infinitely thin and is approximated by the $\delta$-function. The finite thickness $R$ of any realistic mirror provides the physical UV cutoff to the logarithmically divergent integral, $E_{\mathrm{UV}} \sim \hbar c / R$. In many respects the mirror moving in the quantum vacuum is similar to the impurity moving in the condensed matter "vacuum," such as the Fröhlich polaron (electron moving in the vacuum of the phonon quantum field; see review [5]), the Bose polaron (impurity moving in a Bose-Einstein condensate; see Ref. [6] and references therein), a polaron in fermionic "vacuum" (see recent review [7]), and a 
gravitational polaron (see Ref. [8]), etc. However, the model discussed in Ref. [1] has a peculiar property: the interaction between the oscillator and the scalar field is proportional to the time derivative. This is the source of the logarithmically divergent positive contribution to the polaron energy, which adds to the regular negative contribution in the conventional polaron problem and thus reverses the sign of the correction to the mirror mass.

\section{NONRELATIVISTIC MOVING OSCILLATOR}

\section{A. Fröhlich-like description of moving oscillator}

Throughout the text we adopt the system of units with $c=\hbar=1$.

In this section we assume that the thickness of the mirror exceeds its Compton length, $R \gg 1 / M$, where $M$ is the bare mass of the mirror. The classical action for the mirror interacting with the oscillator bound to the mirror and with the massless scalar field can be written in the following form (see Eq. (A1) of Ref. [1]):

$$
\begin{aligned}
S= & \frac{M}{2} \int d t \dot{y}^{2}(t)+\frac{1}{2} \int d x d t\left[\left(\partial_{t} \phi\right)^{2}-\left(\partial_{x} \phi\right)^{2}\right] \\
& +\frac{1}{2} \int d t\left(\dot{q}^{2}(t)-\Omega^{2} q^{2}(t)-2 \epsilon \dot{q}(t) \phi(y[t], t)\right) .
\end{aligned}
$$

Here $y(t)$ is the coordinate of a mirror, $q(t)$ is the variable of the oscillator, and $\phi(x, t)$ is the $1+1$-dimensional scalar field. Here for simplicity we assume that the bare mass $M$ of the mirror is much larger than the ultraviolet cutoff, so that its motion can be considered in the nonrelativistic approximation.

The problem of the mirror moving in the quantum vacuum is in many respects similar to the polaron problem in condensed matter: a single particle interacting with the quantum field of phonons or another bosonic field. As distinct from the conventional polaron problem, in the mirror problem in Ref. [1] the interaction with the scalar field is mediated by the oscillator. The quantization of the classical action Eq. (1) gives rise to the following polaroniclike Hamiltonian:

$$
\begin{aligned}
H= & -\frac{\nabla^{2}}{2 M}+\Omega\left(b^{+} b+\frac{1}{2}\right)+\sum_{q} \omega(q) c_{q}^{+} c_{q}+ \\
& +\epsilon \sum_{q} \frac{\sqrt{\Omega}}{2 \sqrt{\omega(q)}}\left(b-b^{+}\right)\left(c_{q}+c_{-q}^{+}\right) i e^{i q x}+ \\
& +\sum_{q, q^{\prime}} \frac{\epsilon^{2}}{4 \sqrt{\omega(q) \omega\left(q^{\prime}\right)}}\left(c_{q}+c_{-q}^{+}\right)\left(c_{q^{\prime}}^{+}+c_{-q^{\prime}}\right) \\
& \times e^{i\left(q-q^{\prime}\right) x} .
\end{aligned}
$$

Here $b^{+}=\sqrt{\Omega / 2}\left(q-\frac{1}{\Omega} \partial_{q}\right)$ is the creation operator for the oscillator quanta; the second order in the $\epsilon$ term in
Eq. (4) is $\frac{1}{2} \epsilon^{2} \phi^{2}$. It appears due to the transformation $H=L-\dot{q} \partial L / \partial \dot{q}$ from the Lagrangian description in terms of velocity $\dot{q}$ to the Hamiltonian description in terms of momentum $p=\dot{q}-\epsilon \phi=-i \partial_{q}$.

\section{B. Effective mass of the mirror}

In case of a mirror one has two $\epsilon^{2}$ contributions: from the second-order perturbation theory in Eq. (3) with $V_{q}=$ $\epsilon \frac{\sqrt{\Omega}}{2 \sqrt{\omega(q)}}\left(b-b^{+}\right)$,

$$
\begin{aligned}
\Delta \mathcal{E}_{1} & =-\sum_{q} \frac{\left\langle V_{q}^{+} V_{q}\right\rangle}{\Omega+\omega(q)+\frac{q^{2}}{2 M}} \\
& =-\frac{\epsilon^{2} \Omega}{4 \pi} \int_{0}^{\infty} \frac{d q}{\omega(q)} \frac{1}{\Omega+\omega(q)+\frac{q^{2}}{2 M}},
\end{aligned}
$$

and from the first-order perturbation theory for perturbation in Eq. (4):

$$
\Delta \mathcal{E}_{2}=\frac{1}{2} \epsilon^{2}\left\langle\phi^{2}\right\rangle=\frac{\epsilon^{2}}{4 \pi} \int_{0}^{\infty} \frac{d q}{\omega(q)} .
$$

The negative term Eq. (5) corresponds to the conventional correction to the polaronic energy, but now the excited state includes also the first excited level of the oscillator. The positive term Eq. (6) follows from the dependence of the interaction on the time derivative $\dot{q}$.

Thus the total mass of the mirror in the second-order approximation in $\epsilon$ is

$$
M_{\mathrm{eff}}=M+\frac{\Omega}{2}+\epsilon^{2} \int_{0}^{\infty} \frac{d q}{4 \pi \omega(q)}\left(1-\frac{\Omega}{\Omega+\omega(q)+\frac{q^{2}}{2 M}}\right)
$$

In case of a large bare mass of mirror $M \gg \Omega \gg \epsilon^{2}$ and for $\omega(q)=q$, the effective mass of the mirror is:

$$
M_{\mathrm{eff}}=M+\frac{\Omega}{2}+\frac{\epsilon^{2}}{4 \pi} \ln \frac{E_{\mathrm{UV}}}{\Omega},
$$

where $E_{\mathrm{UV}}$ is the UV cutoff. We consider the relativistic spectrum $\omega(q)=q$ of the scalar field, but the nonrelativistic limit for the mirror, i.e., the condition $q^{2} / 2 M \ll$ $q \ll M$. This means that Eq. (8) is valid if

$$
M \gg E_{\mathrm{UV}} \gg \Omega \gg \epsilon^{2} .
$$

Equation (8) coincides with Eq. (111) in Ref. [1] in the limit $\Omega \gg \epsilon^{2}$ with logarithmic accuracy. The nonlogarithmic difference of the order of $\epsilon^{2}$ between the results can be attributed to the fact that we considered the quantum limit for the oscillator. In Ref. [1] the classical equation for the oscillator variable has been used, which is more appropriate in the limit $\Omega \ll \epsilon^{2}$. In this limit Eq. (111) in Ref. [1] gives $M_{\mathrm{eff}}=M+\frac{\epsilon^{2}}{4 \pi} \ln \frac{E_{\mathrm{UV}}}{\epsilon^{2}}$. 
The strong localization of the oscillator in space causes the UV divergence at $k \rightarrow \infty$. To cure this divergence one should consider the more realistic smeared interaction $\epsilon U_{\mathrm{nr}}(x)$ between the mirror and the scalar field instead of the sharp interaction $\epsilon \delta(x)$ in Ref. [1]. The form factor $U_{\mathrm{nr}}(x)$ is localized at the finite distances $\sim R$, which corresponds to the finite width of the mirror. This will be done rigorously in Sec. III. As a result we come to Eq. (8) with the cut off provided by the characteristic length scale of the potential $U_{\mathrm{nr}}(x)$, i.e., $E_{\mathrm{UV}} \sim 1 / R$.

\section{RELATIVISTIC MOVING OSCILLATOR}

\section{A. Field-theoretical description}

Let us start from the action given in [1] for the moving oscillator with mass $M$ moving along the trajectory $y[\tau]$, where $\tau$ is the proper time. The field-theoretical description for this moving object gives the partition function

$$
\begin{aligned}
Z_{1} & =\int D y[\tau] D q[\tau] \exp \left(i \int d \tau\left(-M+\frac{1}{2} \dot{q}^{2}(\tau)-\frac{\Omega^{2}}{2} q^{2}(\tau)\right)\right. \\
& \left.-i \epsilon \int \dot{q}(\tau) \phi(y(\tau)) d \tau\right)
\end{aligned}
$$

where the integral is over the world trajectory of the particle $y$ and over the oscillator coordinates $q$. Let us first work out the integral over $q$. We introduce the annihilation operator

$$
a=\sqrt{\frac{\Omega}{2}}\left(q+\frac{1}{\Omega} \frac{d}{d q}\right) .
$$

Also we introduce the operators $\hat{N}=a^{+} a$ and $\hat{P}=i\left(a-a^{+}\right), \quad$ and the dimensionless constant $\hat{\epsilon}=\epsilon / \sqrt{2 \Omega}$. The standard methods allow to rewrite

$$
\begin{aligned}
Z_{1}= & \int D y(\tau) \operatorname{Tr} P \times \exp \left[-i \int d \tau(M\right. \\
& \left.+\Omega\left(\hat{N}+1 / 2-\hat{\epsilon} \hat{P} \phi(y(\tau))+\hat{\epsilon}^{2} \phi^{2}(y(\tau))\right)\right] .
\end{aligned}
$$

Now partition function $Z_{1}$ describes the particle with the internal discrete degree of freedom $n=0,1,2, \ldots$ We define mass of the moving oscillator at the ground level $|0\rangle: \tilde{M}=M+\Omega / 2$. It takes into account the contribution of the oscillator with $n=0$. The corresponding relativistic field theory is described by the multicomponent spinor $\Psi_{a}$, $a=0,1,2, \ldots$. If the given particle is fermionic, the corresponding partition function receives the form

$$
\begin{aligned}
Z= & \int D \bar{\Psi}(x, t) D \Psi(x, t) D \phi(x, t) \\
& \times \exp \left(\frac{i}{2} \int d x d t\left[\left(\partial_{t} \phi\right)^{2}-\left(\partial_{x} \phi\right)^{2}\right]\right. \\
& +i \int d x d t \bar{\Psi}\left[i \partial_{k} \gamma^{k}-\tilde{M}\right. \\
& \left.\left.-\Omega\left(\hat{N}-\hat{\epsilon} \hat{P} \phi(x)+\hat{\epsilon}^{2} \phi^{2}(x)\right)\right] \Psi\right) .
\end{aligned}
$$

Here the sum is over $k=0,1$, and $\gamma^{0}=\sigma^{1}, \gamma^{1}=i \sigma^{2}$. The Grassmann variable $\Psi$ is multicomponent. In practice we may consider the $K$ component spinor with $K \gg 1$. Coupling constant in this theory is dimensionless. Therefore, the divergences are at most logarithmic.

To check that Eq. (10) indeed corresponds to the second-quantized system with partition function of Eq. (13) let us come to the latter formulation by an alternative way. Namely, let us shift the derivative over $\tau$ in the term $\dot{q}(\tau) \phi(y[\tau])$ of Eq. (10) to $\phi: \dot{q}(\tau) \phi(y[\tau]) \rightarrow$ $-q(\tau) \frac{d}{d \tau} \phi(y[\tau])$. The second-quantized version of the theory for this action looks different from that of given by Eq. (13). It gives the following partition function

$$
\begin{aligned}
Z= & \int D \bar{\Psi}(x, t) D \Psi(x, t) D \phi(x, t) \\
& \times \exp \left(\frac{i}{2} \int d x d t\left[\left(\partial_{t} \phi\right)^{2}-\left(\partial_{x} \phi\right)^{2}\right]\right. \\
& \left.+i \int d x d t \bar{\Psi}\left[\left(i \partial_{k}-\hat{\epsilon} \partial_{k} \phi(x) \hat{Q}\right) \gamma^{k}-\tilde{M}-\Omega \hat{N}\right] \Psi\right) .
\end{aligned}
$$

This looks like the system of spinor field in the presence of a very specific gauge field $\hat{\epsilon} \partial_{k} \phi(x) \hat{Q}$. The action does not contain the interaction term with the second power of the field $\phi$. However, one can prove that this system is indeed equivalent to that of Eq. (13) using the following gauge transformation:

$$
\Psi(x) \rightarrow e^{i \hat{\epsilon} \hat{Q} \phi(x)} \Psi(x) .
$$

\section{B. Mass of the moving oscillator}

Here and below in this subsection we use Euclidean formulation of the model, i.e., assume that the Wick rotation is performed. The one-loop perturbation theory relates the correction to the mass of the moving oscillator $\Delta \mathcal{E}$ with the self-energy function $\Sigma(p)$. Let us calculate this function using the formulation of Eq. (13). We have $\Sigma(p)=\Sigma^{(2)}+\Sigma^{(1)}$ with

$$
\Sigma^{(1)}(p) \approx i \hat{\epsilon}^{2} \Omega \int \frac{d^{2} k}{(2 \pi)^{2}} \frac{1}{k^{2}}
$$


This term corresponds to the one-loop diagram caused by the term $\hat{\epsilon}^{2} \Omega \phi(x)^{2}$. The diagram originated from the term $\hat{\epsilon}^{2} \hat{P}^{2} \phi(x) \phi(y)$ gives

$$
\begin{aligned}
\Sigma^{(2)}(p) \approx & -\hat{\epsilon}^{2} \Omega^{2} \int \frac{d^{2} k}{(2 \pi)^{2}} \\
& \left\langle 0\left|\hat{P} \frac{1}{(p+k) \sigma-i \tilde{M}-i \Omega \hat{N}} \hat{P} \frac{1}{k^{2}}\right| 0\right\rangle .
\end{aligned}
$$

Here $|0\rangle$ is the ground state of the oscillator. The nonrelativistic limit was described in the previous section. It may be obtained here if we suppose that the UV cutoff $\Lambda \ll M$. Then integration over $k^{0}$ gives us the expressions of Sect. II B with $E_{\mathrm{UV}}=\Lambda=1 / R$. Here we consider the opposite limit, when the UV cutoff $\Lambda \gg M$. Since near the pole $p \sigma-i \tilde{M} \sim \epsilon^{2}$, we may set $p^{2}=-\tilde{M}^{2}$ and neglect the terms proportional to $p \sigma-i \tilde{M}$ as these terms result in the renormalization of the propagator and do not contribute to the renormalized mass. The remaining terms are given by

$$
\Sigma^{\prime}(p) \approx i \hat{\epsilon}^{2} \Omega \int \frac{d^{2} k}{(2 \pi)^{2} k^{2}} \frac{k^{2}+\left(2+\frac{\Omega}{\tilde{M}}\right)(k p)}{(p+k)^{2}+(\tilde{M}+\Omega)^{2}}
$$

and are related to the correction to the renormalized mass of the mirror $M_{R}$ as $\Sigma^{\prime}(i \tilde{M}, 0)=i\left(M_{R}-\tilde{M}\right)$. The direct calculation of the integral gives

$$
M_{R}=M+\Omega / 2+\frac{\epsilon^{2}}{4 \pi} \log \left[\frac{\Lambda}{\Omega} \zeta\left(\frac{\Omega}{2 M+\Omega}\right)\right] .
$$

Here

$$
\zeta(x)=\frac{1}{1+\frac{1}{2 x}}\left(1+\frac{1}{4\left(x^{2}+x\right)}\right)^{1+x} .
$$

This function varies between 1 and $1 / 2$. Therefore, at $\Lambda \gg \Omega$ we estimate

$$
M_{R} \approx M+\Omega / 2+\frac{\epsilon^{2}}{4 \pi} \log \frac{\Lambda}{\Omega}
$$

for any relation between $M$ and $\Omega$. This coincides with the nonrelativistic result of Eq. (8). However, in this case we imply that

$$
\frac{1}{R} \gg M, \quad \Omega \gg \epsilon^{2} .
$$

This means that the thickness of the mirror $R$ must be much smaller than its Compton wavelength.

\section{Regularization due to the finite size of the mirror}

Let us introduce the form factor $U(t, x)$ to regularize the UV divergence that comes form the original $\delta$-functional interaction between mirror and scalar field. The corresponding action is given by

$$
\begin{aligned}
S= & \frac{1}{2} \int d^{2} x\left[\left(\partial_{t} \phi\right)^{2}-\left(\partial_{x} \phi\right)^{2}\right] \\
& +\int d^{2} x \bar{\Psi}\left[i \partial_{k} \gamma^{k}-\tilde{M}-\Omega \hat{N}\right] \Psi \\
& +\Omega \hat{\epsilon} \int d^{2} x \bar{\Psi}(x) \hat{P} \Psi(x) \int d^{2} z U(x-z) \phi(z) \\
& -\Omega \hat{\epsilon}^{2} \int d^{2} x \bar{\Psi}(x) \Psi(x)\left(\int d^{2} z U(x-z) \phi(z)\right)^{2} .
\end{aligned}
$$

The model considered in Ref. [1] is restored in the infinitely thin limit $U(x, t)=\delta(x) \delta(t)$. One can see, that the effect of the form factor $U$ may be taken into account via the renormalization of the scalar field propagator:

$$
\frac{1}{k^{2}} \rightarrow U(k) \frac{1}{k^{2}} U(-k) .
$$

Here $U(k)=U\left(k_{0}, k_{1}\right)$ is the Fourier transform of $U(t, x)$. The limit of the infinitely thin mirror appears when $U(k)=1$. In the nonrelativistic case we should consider $U(t, x)=U_{\mathrm{nr}}(x) \delta(t)$ and assume, that $U_{\mathrm{nr}}$ is real valued. In the general case of relativistic-invariant theory the situation is more involved. The form factor $U$ (that may be complex valued) should depend on the invariant interval $s^{2}=t^{2}-$ $x^{2}$ and decreases fast at $\left|s^{2}\right| \rightarrow \infty$.

In nonlocal relativistic quantum field theory the finite size of the source may be taken into account using the form factors in momentum space that depend on the invariant $p^{2}$ (see [9] and references therein). At least for the static sources of the finite size, such form factors indeed model the field caused by the charge distributed over the finite region of space. Below we consider the particular form of $U$ that is caused by the interaction with an intermediate auxiliary field $\theta$ of mass $\Lambda$. A pointlike mirror emits/ absorbs one or two quanta of the field $\theta$. The vertexes for the emission/absorption of $\theta$ are the same as those for the emission/absorption of $\phi$ in Eq. (13). The quanta of the field $\theta$ may be transformed to the quanta of the field $\phi$. We know that the exchange by massive particles in field theory describes the interaction that occurs at finite distances. This finite distance may be evaluated as $R=1 / \Lambda$. That's why we consider the exchange by massive particle as the device for modeling the finite size of the mirror. This leads us to the interpretation of $U$ as the propagator of $\theta$ (up to the normalization constant) while Eq. (23) is the effective action of the theory obtained after the field $\theta$ is integrated out. This results in the following form of the function $U(k)$ in momentum space:

$$
U(k)=-\frac{\Lambda^{2}}{k^{2}-\Lambda^{2}+i 0} .
$$


The corresponding function $U(t, x)$ in $1+1$-dimensional coordinate space is expressed through the complexvalued special functions. One can check, that the absolute value of $U(t, x)$ falls sharply at $\left|t^{2}-x^{2}\right| \Lambda^{2} \rightarrow \infty$. For the renormalized mass of the mirror we have

$$
\begin{aligned}
M_{R}= & M+\Omega / 2 \\
& +\hat{\epsilon}^{2} \Omega \int \frac{d^{2} k}{(2 \pi)^{2} k^{2}} U(k) U(-k) \frac{k^{2}+\left(2+\frac{\Omega}{\tilde{M}}\right)(k p)}{(p+k)^{2}+(\tilde{M}+\Omega)^{2}} .
\end{aligned}
$$

Here the integral is over the Euclidean 2-momentum a $k$, while $U(k)=\frac{1}{1+k^{2} R^{2}}$ is obtained from $U\left(k_{0}, k_{1}\right)$ by analytical continuation. Then one can easily derive (at $\Omega R \ll 1)$,

$$
M_{R} \approx M+\Omega / 2+\frac{\epsilon^{2}}{4 \pi} \log \frac{1}{\Omega R}
$$

\section{CONCLUSION}

We suggest the description of the model for the $1+1$ dimensional mirror discussed in Ref. [1] that reveals its analogy to polaron. We consider the field theory that contains the multicomponent spinor field interacting with the scalar field. This is the second quantized theory that describes moving oscillator interacting with the scalar field. Actually, this model may easily be formulated in the spacetime of any dimension. Being defined in the $3+1$ dimensional spacetime it may have certain applications in the high-energy physics if it is necessary to describe the fermionic particle with an infinite (or large) number of internal energy levels. (For example, in [10] it is suggested, that such an internal degree of freedom marks the flavor of the Standard Model fermion.) However, here we restrict ourselves by the consideration of the 1+1-dimensional case that exactly matches the model of [1]. In this case the moving oscillator may be considered as a model of the moving mirror.

We consider the two versions of the second-quantized theory of the mirror. The two formulations are exactly equivalent as follows from our analysis. The formulation of Eq. (13) reveals the analogy with the usual polaron problem. At the same time the formulation of Eq. (15) contains the interaction of the mirror with the special gauge field composed of the scalar excitations. We suppose that this formulation may be useful for the further applications of the developed formalism to various problems both in the high energy physics and in the condensed matter physics.

We demonstrate that in the discussed model there appears the extra term [Eqs. (6), (16)] in the energy of the polaron, which is logarithmically divergent. This extra term reverses the sign of the energy correction as compared to the negative mass correction in the conventional polaron problem. The obtained infinite value of the mirror mass is the artifact of the model, which uses the artificial $\delta$-function potential. The logarithmically divergent integral is regularized by any realistic potential which provides the natural UV cutoff. In this mirror-polaron problem, the UV divergence is the physical effect, while different magnitudes $E_{\mathrm{UV}}$ of the UV cutoff reflect different physical mechanisms.

\section{ACKNOWLEDGMENTS}

The work of M.A.Z. is supported by the Natural Sciences and Engineering Research Council of Canada. G. E. V. acknowledges financial support from the Academy of Finland and its COE program.
[1] Qingdi Wang and W. G. Unruh, Phys. Rev. D 89, 085009 (2014).

[2] G. E. Volovik, JETP Lett. 77, 639 (2003).

[3] F. R. Klinkhamer and G. E. Volovik, JETP Lett. 91, 259 (2010).

[4] G. E. Volovik, Phys. Rev. Lett. 46, 1351 (1981)

[5] J. T. Devreese and A. S. Alexandrov, Rep. Prog. Phys. 72, 066501 (2009).
[6] Weiran Li and S. Das Sarma, Phys. Rev. A 90, 013618 (2014).

[7] Zhihao Lan and Carlos Lobo, J. Indian Inst. Sci. 94, 179 (2014).

[8] A. Comech and M. Zubkov, J. Phys. A 46, 435201 (2013).

[9] G. V. Efimov, Nonlocal interactions of quantum fields (Nauka, Moscow, 1977).

[10] Bodo Lampe, Mod. Phys. Lett. A 28, 1350018 (2013). 